\title{
Simultaneous Penetrating Keratoplasty with Crescentic Inferior Keratoplasty in Advanced Pellucid Marginal Degeneration
}

\author{
${ }^{1}$ Valentine Saunier, ${ }^{2}$ David Smadja, ${ }^{3}$ David Touboul
}

\begin{abstract}
Purpose: To describe and report long-term outcomes of a surgical technique in advanced pellucid marginal degeneration (PMD) combining conventional central penetrating keratoplasty (PK) with an additional crescentic inferior keratoplasty, using a single corneal transplant.
\end{abstract}

Results: We report the case of a 55-year-old male patient, who underwent a customized sizing PK in his right eye for an advanced PMD, which combined a central PK with an additional crescentic inferior keratoplasty. The 1 year postoperative results show very good clinical outcomes, including visual acuity and biomechanical parameters, as well as tectonic outcomes, documented with high resolution optical coherence tomography (OCT) at the junction site between the two grafts.

Conclusion: Advanced PMD is a specifically challenging condition for keratoplasty due to an extreme peripheral corneal thinning. This surgical technique may facilitate inferior suturing and optimize the postoperative tectonic outcomes.

Keywords: Crescentic keratoplasty, Double keratoplasty, Pellucid marginal corneal degeneration, Penetrating keratoplasty.

How to cite this article: Saunier V, Smadja D, Touboul D. Simultaneous Penetrating Keratoplasty with Crescentic Inferior Keratoplasty in Advanced Pellucid Marginal Degeneration. Int J Kerat Ect Cor Dis 2015;4(2):60-62.

Source of support: Dr Smadja is a paid consultant for Alcon laboratories (Fort Worth, US) and Ziemer System.

Dr Touboul is a paid consultant for Moria Surgical (Antony, France) and Horus pharma (Saint-Laurent du Var, France). The rest of the authors have no financial interest.

Conflict of interest: None

\footnotetext{
${ }^{1}$ Resident, ${ }^{2}$ Clinician, ${ }^{3}$ Professor

${ }^{1,3}$ Department of Ophthalmology, Anterior Segment Unit Bordeaux Hospital University, National Reference Center for Keratoconus, Bordeaux, France

${ }^{2}$ Department of Ophthalmology, Anterior Segment Unit Bordeaux Hospital University, National Reference Center for Keratoconus, Bordeaux, France; Institute of Nanotechnologies and Advances Materials, Bar Ilan University, Ramat Gan, Israel

Corresponding Author: Valentine Saunier, Resident Department of Ophthalmology, University Center Hospital of Bordeaux, Anterior Segment and Refractive Surgery Unit; National Reference Center for Keratoconus, Place Amélie Rabat-Leon 33000, Bordeaux, France, Phone: +33623933461 e-mail: valentine.saunier@chu-bordeaux.fr
}

\section{INTRODUCTION}

Pellucid marginal degeneration (PMD) corneal is a rare, non-inflammatory and bilateral corneal ectasia, clinically characterized by a peripheral inferior area of stromal thinning that occurs from 1 to $3 \mathrm{~mm}$ from the limbus in the 4 to 8 o'clock meridians. ${ }^{1,2}$ This rare ectatic condition may progressively lead to significant corneal asymmetry with high irregular astigmatism that ultimately impairs the vision. According to the severity of this astigmatism, multiple options may be considered to recover a satisfying visual acuity and corneal transplant has been proposed as a relatively effective solution in the most advanced cases of PMD. ${ }^{1,2}$ However, surgical correction of this condition still remains a challenge in view of the peripheral and asymmetrical nature of the corneal thinning, which generally limits the completion of conventional central lamellar or penetrating keratoplasty (PK). A variety of surgical techniques have already been tried, including wedge resection, $\mathrm{PK}$, inferior eccentric PK, central lamellar keratoplasty, crescentic wedge resection, crescentic lamellar keratoplasty and simultaneous peripheral crescentic lamellar keratoplasty with central PK with varying degrees of success. ${ }^{3}$

With this case, we report and illustrate a potential surgical alternative to conventional central PK in order to overcome intraoperative suture-related complications and to optimize postoperative tectonic outcomes. This technique consists in a simultaneous PK with crescentic inferior keratoplasty using a single donor. One year postoperative clinical (visual acuity, biomechanical parameters and endothelial cell count) and tectonic outcomes (OCT and confocal microscopy at the junction site between the two graft tissues) are reported and discussed herein.

\section{CASE REPORT}

A 55-year-old male was referred to the Department at the Keratoconus National Reference Center, in Bordeaux Hospital University, France, for a progressive decrease in vision in both eyes reported over the past 3 years. Ocular history included a diagnosis of PMD made 5 years earlier with no other ocular conditions, and rigid contact lens 

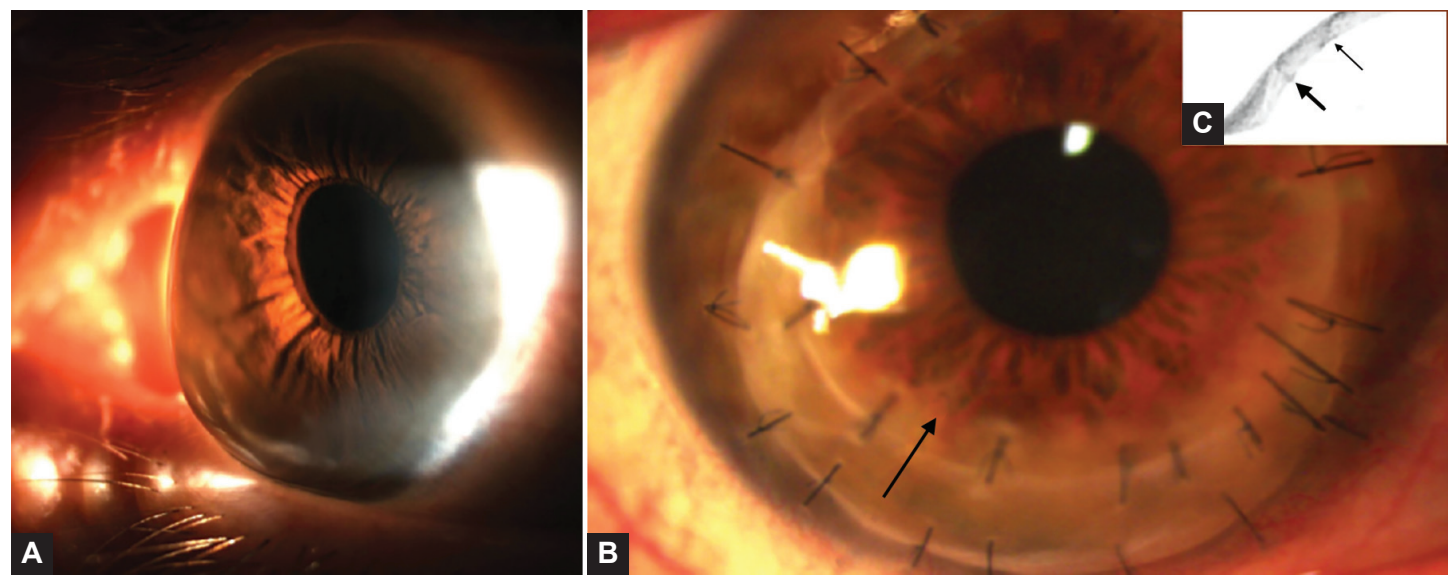

Figs 1A to C: (A) Preoperative examination on right eye shows the inferior thinning and the extreme corneal protrusion, (B) slitlamp examination at 8 month's postoperative. The graft is clear and centered on the pupil. The arrow shows the section of the OCT, (C) 8 months postoperative OCT. The crescent is recessed between the inferior edge of the central button (thin arrow) and the patient's cornea (thick arrow)

intolerance due to a very advanced stage of PMD with a mild dry eye syndrome.

On examination, his best spectacles corrected visual acuity (BSCVA) was 20/200 and 20/50 with $+4(-6)$ at $140^{\circ}$ and $+1(-10)$ at $80^{\circ}$, respectively in the right and left eye. The slit-lamp examination confirmed a bilateral inferior and peripheral corneal thinning with no corneal infiltration or neovascularization (Fig. 1A). Corneal topography showed an inferior corneal steepening in a butterfly pattern in both eyes with a maximal keratometry and keratometric astigmatism of $66.8 \mathrm{D}$ and $24.6 \mathrm{D}$ on the right eye (Table 1) and 55.7 D and 13.2 D on the left eye. Thinnest points measured with Visante OCT (Carl Zeiss Meditec) were $273 \mu$ and $350 \mu$, respectively in the right and left eye. Corneal transplant with simultaneous central PK combined to inferior crescentic keratoplasty was decided for the right eye, due the very advanced stage of the disease, whereas intrastromal corneal rings were first planned on the left eye.

The surgery was performed under general anesthesia and corneal dissection was performed according to the following illustrated steps (Figs 2A to F). The central donor cornea was first trephined with an $8.25 \mathrm{~mm}$ diameter Hessburg-barron trephine (Katena Products Inc) (Fig. 2A). Then, a full-thickness crescent was removed to the donor graft based on the distance measured on the patient cornea to fill the gap in the thinnest part of the inferior cornea (Fig. 2B). Patient's central cornea was then trephined with a slightly lower diameter (8 $\mathrm{mm}$ trephine) (Fig. 2C) and the initial $8.25 \mathrm{~mm}$ donor's trephine was used to make an imprint inferiorly on the corneal recipient through the epithelium without penetrating the cornea, to mark the corresponding crescentic area to be dissected manually (Fig. 2D). The central donor graft was first sutured with eight interrupted Nylon 10.0 sutures (Fig. 2E) while the crescentic keratoplasty was secondary sutured inferiorly to fill the gap (Fig. 2F) with 14 sutures.

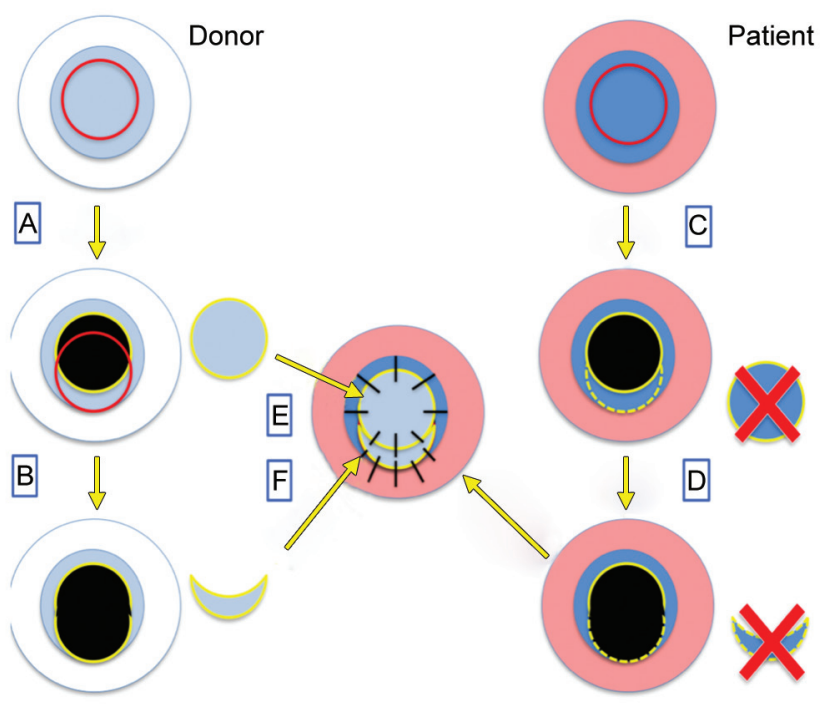

Figs 2A to F: Schema of the surgical technique. The different steps were described in the manuscript

Postoperatively, no early complications were noted, such as corneal infection, epithelial healing delay, acute rejection or sutures-related complications. At 8 months, the graft was clear and centered on the pupil (Fig. 1B). At 1 year, the BSCVA was 20/25 with $-1.25(-2.25)$ at $60^{\circ}$ in the right eye and $20 / 25$ with $-1.25(-2.50)$ at $90^{\circ}$ in the left eye, after the intracorneal ring implantation. On the topography, keratometric astigmatism and maximal keratometry in the right eye were $4.1 \mathrm{D}$ and $47.2 \mathrm{D}$ respectively (Table 1). The high resolution OCT (Visante OCT Carl Zeiss Meditec) showed the very good match

Table 1: Keratometric astigmatism, maximal keratometry and BSCVA at baseline, 6,12 and 24 months postoperatively

\begin{tabular}{lllll}
\hline & Preoperative & M6 & M12 & M24 \\
\hline $\mathrm{K}_{\max }$ (D) & 66.8 & 49.1 & 47.2 & 44.2 \\
Cyl (D) & 24.6 & 6.2 & 4.1 & 2.9 \\
BSCVA & $20 / 200$ & $20 / 40$ & $20 / 25$ & $20 / 25$ \\
\hline
\end{tabular}

Legends: K: Keratometry max; Cyl: Keratometric astigmatism (cylinder); BSCVA: Best spectacle corrected visual acuity 
of the corneal wound between the two corneal buttons (Fig. 1C). The postoperative progression of the BSCVA and corneal astigmatism over a 2-year follow-up period was summarized in Table 1.

\section{DISCUSSION}

Pellucid marginal degeneration is a non-frequent noninflammatory corneal dystrophy sometimes related to keratoconus with unknown pathophysiology. The absence of treatment can lead to acute hydrops (2.5 to $6 \%$ of cases) and perforation as a result of progressive thinning. ${ }^{1,2}$ Further current options in the surgical management of PMD have been described: corneal collagen cross-linking, intracorneal ring implantation, tuck-in lamellar keratoplasty, crescentic lamellar keratoplasty, crescentic lamellar wedge resection, deep anterior lamellar keratoplasty (DALK), full-thickness crescentic wedge resection and PK. ${ }^{3}$ The most common surgical treatments of PMD are PK and DALK.

Because of the location of thinning area, conventional technique of PK requires large-diameter grafts increasing the risk of corneal vascularization, limbal stem cell deficiency, wound dehiscence and graft rejection. Decentered grafts also work poorly because of large degree of astigmatism caused by the decentering and a higher incidence of rejection resulting from the proximity to the limbus. Indeed, Varley et $\mathrm{al}^{4}$ reported at least one endothelial rejection episode in seven of 11 (64\%) corneal transplants. Moreover, large or decentered grafts are responsible for higher incidence of angle damage and peripheral anterior synechiae thus causing raise of intraocular pressure.

Deep anterior lamellar keratoplasty benefits are a reduced endothelial cell loss and a limited risk of graft rejection. Nevertheless, suturing inferiorly the corneal button on a very thin recipient wedge remains challenging. Thus, this procedure required an experimented operator because of higher rate of conversion to PK than in keratoconus. To illustrate, Al-Torbak published a series with 16 eyes affected of DMP requiring a DALK, 12 perforations have been reported. ${ }^{5}$ Rasheed and Rabinowitz ${ }^{6}$ described a technique which combine simultaneous central PK with peripheral crescentic lamellar keratoplasty to reinforce the area of thinning followed by selective suture removal and astigmatic keratotomy in the postoperative period. The five patients of this study had a BSCVA after rehabilitation period between 20/70 and $20 / 25$. This results were encouraging but a longterm follow-up is required to determine the incidence of rejection of the central penetrating graft. MacLean et $\mathrm{al}^{7}$ reported their results in 10 eyes treated with corneal wedge excision for PMD. They obtained good results in terms of time required for visual rehabilitation and the reduction in corneal astigmatism. However, in all eyes treated were noted a progressive increase in against the rule astigmatism and the refraction at the follow-up period seemed unstable. In our technique, the crescent strengthens and thickens the inferior area of cornea and makes the suturing easier peroperatively. Finally, it seems to be less technically complex than DALK and could decrease the risk of complication comparing with conventional PK. Good outcomes were achieved for this patient within the 2 years of postoperative follow-up in terms of time required for visual rehabilitation, refractive results, corneal astigmatism and tectonically results. Indeed, the patient achieved visual acuity of 20/40 at 6 months and 20/25 at 12 months. The refraction and the corneal astigmatism was stable over the 2 years. A long-term follow-up period is necessary for evaluate the risk of cornea rejection. No definite guideline exists for the management of advanced PMD. In our experience, PK by using an inferior crescent to tectonically maintain the graft offers an effective and safe option.

\section{REFERENCES}

1. Sridhar MS, Mahesh S, Bansal AK, Nutheti R, Gao GN. Pellucid marginal corneal degeneration. Ophthalmol 2004; 111(6):1102-1107.

2. Krachmer JH. Pellucid marginal corneal degeneration. Arch Ophthalmol 1978;96(7):1217-1221.

3. Moshifar M, Edmonds J, Behunin N, Christiansen S. Current options in the management of pellucid marginal degeneration. J Refract Surg 2014;30(7):474-485.

4. Varley GA, Macsai MS, Krachmer JH. The results of penetrating keratoplasty for pellucid marginal corneal degeneration. Am J Ophthalmol 1990;110(2):149-152.

5. Al-Torbak AA. Deep anterior lamellar keratoplasty for pellucid marginal degeneration. Saudi J Ophthalmol 2013; 27(1):11-14.

6. Rasheed K, Rabinowitz YS. Surgical treatment of advanced pellucid marginal degeneration. Ophthalmol 2000;107(10): 1836-1840.

7. MacLean H, Robinson LP, Wechsler AW. Long-term results of corneal wedge excision for pellucid marginal degeneration. Eye (Lond) 1997;11(Pt 5):613-617. 